\title{
THE CLINICOPATHOLOGICAL ROLES OF ALPHA B-CRYSTALLIN AND P53 EXPRESSION IN PATIENTS WITH HEAD AND NECK SQUAMOUS CELL CARCINOMA
}

\author{
Karin Boslooper ${ }^{1}$, medical student \\ Alfred King-Yin Lam², MBBS, PhD, MD, FRCPA, \\ Jin $\mathrm{Gao}^{1}$, BDS, MSc, PhD, \\ Stephen Weinstein ${ }^{2}$, MBBS, FRCPA, \\ Newell Johnson ${ }^{1}$, BDSc, MDSc, PhD, FRCPath \\ ${ }^{1}$ School of Dentistry and Oral Health, Griffith University \\ ${ }^{2}$ School of Medicine, Griffith University \\ Acknowledgements: The authors would like to thank the staff in Queensland Pathology in \\ providing access to slides and blocks for the study, Dr Robert Smith from Griffith Medical \\ School in providing some of the laboratory support and Dr Lis Sedlak-Weinstein for \\ reviewing the manuscript. The study was supported by a research fund of School of Dentistry \\ and Oral Health, Griffith University.
}

Meeting Presentation: An abstract of the paper was presented in the First World Congress of the International Academy of Oral Pathology, 2007 at Amsterdam

Running title: p53 and alpha-B-crystallin in head and neck cancer

Key words: head and neck, squamous cell carcinoma, heat shock protein, alpha B-crystallin, p53

\section{Correspondence/reprint request:}

Professor Alfred Lam

Griffith Medical School

GH1, Medicine and Oral Health Centre

Gold Coast Campus

Gold Coast QLD 4222

Australia

Phone: +61 756780718

Fax: +61 756780708

E-mail: a.lam@griffith.edu.au 


\section{ABSTRACT}

Aims: The aim of the study was to investigate the expression of alpha-B-crystallin and p53 in HNSCC.

Methods: Alpha-B-crystallin and p53 expressions from 118 HNSCC were studied by immunohistochemistry and correlated with clinicopathological parameters.

Results: Alpha-B-crystallin expression was seen in 28\% ( $n=33)$ of HNSCC. All, except one, of poorly-differentiated HNSCC were negative for alpha-B-crystallin. P53 expression was seen in $63 \%(n=73)$ of HNSCC and was more common in moderately/poorly-differentiated HNSCC $(p=0.034)$. The proportion of cases with positive staining for either alpha-Bcrystallin or p53 was different in different anatomical locations in head and neck. Patients with HNSCC having a high portion of tumour cells expressing p53 had a shorter survival than the other groups $(\mathrm{p}=0.032)$.

Conclusion: The expression of p53 and alpha-B-crystallin were related to the differentiation and site of the HNSCC. Alpha-B-crystalline was not a prognostic marker for HNSCC. 


\section{INTRODUCTION}

Head and neck squamous cell carcinoma (HNSCC) is the sixth most common cancer worldwide with a global annual incidence of $500,000 .{ }^{1} \mathrm{HNSCC}$ is a heterogeneous group of cancers, with a variable, but usually poor prognosis in patients. ${ }^{1}$ Despite advances in treatment, the mortality rate of HNSCC has not changed markedly over the last few decades. ${ }^{2}$ Besides the poor outcome, head and neck cancer has a great impact on the patient's quality of life, due to its anatomic location. The cancer treatment involves pain and disfigurement and it can affect speech, breathing and swallowing. ${ }^{3}$ Clinicopathological criteria are important for the management and prediction of outcome of patients. However, within a group of patients sharing the same features, there is still significant variation in prognosis. Studies on the carcinogenesis in HNSCC can lead to understand its biological behaviour and to reveal the underlying mechanisms of cancer progression and therapy resistance. ${ }^{4}$

The tumour suppressor gene $p 53$, located on the short arm of chromosome 17, is known as 'the guardian of the genome'. Its key function is the control of cell cycle. P53 has the ability to detect either physical or chemical DNA damage. In response, by acting as a transcription factor, it activates other genes necessary for cell cycle arrest; thereby allowing DNA-repair before duplication. If damage to DNA is too severe, the cell will undergo apoptosis. Mutation of the p53 gene is the most common mutation in human cancer; $40-50 \%$ of HNSCC carry a p53 mutation. ${ }^{6}$ Inactivation of p 53 will deregulate this control function in the cell cycle. Despite the presence of DNA damage, the cell will continue its replication instead of undergoing apoptosis. This contributes to genomic instability and makes the cell relatively resistant to chemo- and radiotherapy. ${ }^{5,7}$ The wild type $\mathrm{p} 53$ protein has a short halflife and cannot be detected by immunohistochemistry. The mutated p53 protein usually has a long half-life and therefore can be detected by immunohistochemistry. 
Alpha-B-crystallin is a member of small heat-shock proteins and has a cytoprotective function. Under physiologic conditions, the level of small heat-shock proteins in tissues is low. ${ }^{8}$ One exception is in the eye lens where alpha-B-crystallin accounts for 40 percent of the cytoplasmic proteins and plays a role in maintaining its transparency. ${ }^{9}$ Alpha-B-crystallin is elevated under physiologic stress, neurodegenerative diseases and cancer, eg gliomas, renal carcinomas and breast carcinomas. ${ }^{9}$ Alpha-B-crystallin acts as a molecular chaperone, by preventing aggregation of denatured proteins after exposure to stresses such as heat shock, radiation, oxidative stress and anticancer drugs. ${ }^{10}$ Furthermore, it has a strong anti-apoptotic effect. Alpha-B-crystallin interacts with different apoptotic proteins at key regulatory points in the control of apoptosis. ${ }^{8}$ It inhibits the proteolytic activation of caspase- $3^{11}$ and binds to pro-apoptotic Bcl-2 family members, Bax and $\mathrm{Bcl}-\mathrm{x}_{\mathrm{s}}{ }^{10}$, thereby preventing their translocation to the mitochondria. It has also been shown that alpha-B-crystallin protects cells from death induced by proteasome inhibition. ${ }^{8}$ The role of alpha-B-crystallin in these apoptotic pathways can be an important mechanism in therapy resistance. Kamradt et al in 2005 reported alphaB-crystallin is an inhibitor of TRAIL (tumour necrosis factor-related apoptosis-inducing ligand)-induced apoptosis, which is a promising anti-tumour reagent. ${ }^{12}$

As both P53 and alpha-B-crystallin play roles in apoptotic pathways in tumourigenesis, the aim of this study is to evaluate these roles in Australian patients with head and neck squamous cell carcinomas. This is the largest series for the study of alpha-Bcrystalline in HNSCC. 


\section{MATERIAL AND METHODS}

\section{Patients and tumour tissues}

Between September 1997 and December 2005, 208 patients were diagnosed with head and neck squamous cell carcinoma at the Gold Coast Hospital in Queensland, Australia. In this study, patients with cancers of lips and oesophagus were excluded from the study. The pathology of the other patients was reviewed by the authors (KB and AKYL) according to the World Health Organization of tumours. ${ }^{13}$ After the review, paraffin blocks from these patients were chosen for immunohistochemical study. In the selection of the paraffin blocks, normal tissue and dysplastic mucosa was included, whenever possible, with the tumour.

One hundred and eighteen patients ( 31 women and $88 \mathrm{men}$ ) with a mean age of 62 were selected. Of these, $34.7 \%(n=41)$ of the SCC were located in the oral cavity, $27.1 \%$ $(n=32)$ in the oropharynx, $23.7 \%(n=28)$ in the larynx, $14.4 \%(n=17)$ in the hypopharynx. The archival cancer tissue blocks were provided by Queensland Health Pathology Service at Gold Coast Hospital and the patient's clinical and survival data were obtained from the patient charts. Ethic approval was given by both Human Research Ethic Committees of Gold Coast Hospital and Griffith University.

\section{Immunohistochemistry}

Formalin-fixed and paraffin embedded tissue blocks were cut at $5 \mu \mathrm{m}$ and placed on coated slides. The sections were dewaxed in xylene and rehydrated in ethanol. For p53 analysis the sections were incubated in BORGdecloaker $(\mathrm{pH}=9)$ (Biocare Medical, Concord, Australia) and then immediately heated in a pressure cooker at $125^{\circ} \mathrm{C}$ for four minutes for antigen retrieval. A monoclonal antibody (P53-DO7, Novocastra Laboratories, Newcastle, UK), at a 1:200 dilution, was applied to the sections and incubated overnight at $4{ }^{\circ} \mathrm{C}$. For alpha-B-crystallin analysis, the sections were incubated in a $10 \mathrm{mM}$ citrate acid buffer $(\mathrm{pH}$ 
$=6$ ) and heated at $121^{\circ} \mathrm{C}$ for five minutes for antigen retrieval. A monoclonal antibody (SPA222, Stressgen bioreagents, Michigan, USA), at a 1:200 dilution, was applied and sections were incubated overnight at room temperature.

For both reactions, the DAKO EnVision Detection System (Gloustrup, Denmark) was used to increase the sensitivity of the experiment, followed by the colour development, which was performed with diaminobezidine (DAB) incubated for 5 minutes. The counterstaining with Mayer's hematoxylin was performed for morphology. A case, with strong positive staining, was included in each series of immunostaining to act as a positive control. For a negative control, the primary antibody was replaced by $2 \%$ Bovine Serum Albumin/Phosphate Buffered Saline (BSA/PBS).

Evaluation of the immunostains was done by the authors [KB \& AL]. The expressions of p53 and alpha-B-crystallin were evaluated by the percentage of positively stained cells, with a division into four grades: grade 0 (0 -9\%), grade 1 (10-29\% positive), grade 2 (30-69\% positive) and grade 3 (70-100\% positive). For p53, nuclear staining was regarded as positive whereas for alpha-B-crystallin, cytoplasmic staining was regarded as positive.

\section{Statistical analysis}

Categorical data was analyzed with either the chi-square test or the Fisher's exact test, depending on the number of cases. Disease-free survival (time between diagnosis of primary tumour and recurrence) and overall survival (time between diagnosis of primary tumour and death) were calculated using the Kaplan-Meier method. The log rank test was used to compare the survival curves of different groups. A P-value $(p<0.05)$ was considered as statistically significant. Data analysis was performed using SPSS (Statistical Package for the Social Sciences for Windows, version 14.0, Chicago, IL, USA) 


\section{RESULTS}

\section{Pathological features}

Thirty-five percent $(n=41)$ of the cancers were classified as well differentiated, $56 \%$ $(n=66)$ as moderately differentiated and $9 \%(n=11)$ as poorly differentiated. According to the TNM classification $23 \%(n=27)$ were diagnosed as stage I, 20\% $(n=24)$ as stage II, $16 \%$ $(n=19)$ as stage III and $41 \%(n=48)$ as stage IV. At the time of diagnosis $42 \%(n=50)$ of the patients had a positive nodal status.

\section{P53 immunoreactivity}

P53 positivity was seen as nuclear staining. P53 immunoreactivity was observed in $63 \%(n=73)$ of the tumours, of which 29 cases were classified as grade 1, 20 cases as grade 2 and 24 cases as grade 3 . In well and moderately differentiated squamous cell carcinomas, p53 positive staining cells were mainly located in periphery of the tumour cell nests (Figure 1). Positive staining was also seen in some dysplastic lesions. The non-tumour tissue was negative for $\mathrm{p} 53$.

The relationship between $\mathrm{p} 53$ positivity and the clinicopathological parameters were shown in Table 1. P53 immunoreactivity correlated with the differentiation of the tumour. It is more often noted in higher grade lesions. Also, grade two and grade three p53 positivity were seen more frequently in moderately and poorly differentiated squamous cell carcinomas (Chi-square test, $\mathrm{p}=0.034$ ). The prevalence of $\mathrm{p} 53$ expression differed between the different sites. P53 positive staining was less frequently observed in tumours from the larynx (50\%; $n=14 / 28)$, in comparison with the oropharynx $(66 \% ; n=21 / 32)$, hypopharynx $(65 \% ; n=11 / 17)$ and the oral cavity $(66 \% ; n=27 / 41)$ although not statistically significant $(\mathrm{p}=0.182)$. There was no correlation found between p53 positivity with gender, age, TNM stage and the event of recurrence. 


\section{Alpha-B-crystallin immunoreactivity}

The alpha-B-crystallin positivity was seen as cytoplasmic staining. Alpha-Bcrystallin expression was seen in $28 \%(n=33)$ of the HNSCC, of which 19 cases classified as grade one, 10 cases as grade two and 4 cases as grade three. In well and moderately differentiated HNSCC, the positive staining was predominately located in the cells in the centre of the tumour cell nests (Figure 2). Few dysplastic lesions showed positive staining for alpha-B-crystallin. Positive staining was also seen in muscle, salivary glands, blood vessels and in some stromal cells. All, but one of the poorly-differentiated HNSCCs were negative for alpha B-crystallin. Alpha-B-crystallin positive staining was more often seen in the hypopharynx $(35 \% ; n=6 / 17)$ and the oral cavity $(34 \% ; n=13 / 41)$, followed by the larynx $(29 \% ; n=8 / 28)$ and the oropharynx $(19 \% ; n=6 / 32)$. There was no correlation found with gender, age, TNM stage and the event of recurrence (Table 2).

\section{Relationship between p53, alpha-B-crystallin}

Twenty-eight per cent $(n=33)$ of the tumours were negative for both p53 and alpha-Bcrystallin and $18 \%(\mathrm{n}=21)$ were positive for both. There was no correlation noted between p53 and alpha B-crystallin expressions.

\section{Survival analysis}

At the time of analysis, 59 patients were alive, 42 had died and 17 patients had no adequate follow-up data. Patients with no adequate follow-up data were excluded from the survival analysis. The median follow-up time for the remaining group was 24.6 months, with a 5-year survival of $30 \%$. The group of patients who were alive at the last date of follow-up had a median follow-up time of 31.4 months. Recurrence of the cancer was reported in 36 
patients and diagnosed within a period of 1 to 51 months, with a median of 9 months. Patients diagnosed with stage four cancer had a significantly shorter disease free survival $(p=0.036)$ compared with stage one, two and three. Patients with a positive nodal status also had a shorter disease free survival $(\mathrm{p}=0.019)$ compared with negative nodal status. The patients with a grade three p53 expression of the tumour had a significantly shorter overall survival time $(\mathrm{p}=0.032)$ in comparison with grade one and grade two p53 expression (Figure 3). Between the different groups of alpha-B-crystallin expression there was no significant difference found in both disease-free and overall survival. 


\section{DISCUSSION}

The tumour suppressor gene $p 53$ is involved in the regulation of heat shock protein (HSP) in cancer, by repressing the transcription of the heat shock protein genes. If $p 53$ mutation is present in tumour cells, this leads to an increase of transcription of these genes. ${ }^{14}$ Recently, it was shown that alpha-B-crystallin binds to p53 to sequester its translocation to mitochondria during hydrogen peroxide-induced apoptosis. ${ }^{15}$ In this study, involving a large cohort of patients, no significant correlation between the expression of p53 and alpha-Bcrystallin was found in HNSCC. It is likely that the correlations between these molecules are more complex.

In this study, the expressions of alpha-B-crystallin were noted to be associated with the tumour cell differentiation. The expression of alpha-B-crystallin was inversely correlated with differentiation; all, except one, of the poorly differentiated tumours were negative for alpha-B-crystallin. The inverse correlation between differentiation and the expression of alpha-B-crystallin has not been described by previous studies on alpha-B-crystallin. Also, the alpha-B-crystallin was noted in the central well-differentiated portion of the HNSCC. This observation has not been described in HNSCC in the literature. This may provide evidence that the well-differentiated HNSCC are more resistance to apoptosis.

In oral squamous cell carcinomas, P53 expression has been related to the grade of differentiation. ${ }^{16,17}$ In this study, p53 expression was also correlated to tumour grade with high percentages of staining more frequently observed in moderately and poorly differentiated carcinomas. In addition, nuclear staining of p53 was noted more frequently in the peripheral portion of the tumour cell nests which is the more poorly-differentiated portion of the tumour.

Both $\mathrm{p} 53$ and alpha-B-crystallin showed a variance in expression among the different sites. P53 was less frequently observed in the larynx, in comparison with oropharynx, 
hypopharynx and oral cavity. For alpha-B-crystallin, the expression was more often seen in hypopharynx and oral cavity, followed by larynx and oropharynx. This finding suggests a difference in molecular pathways of carcinogenesis among the different sites as the different exposure, resistance to carcinogens and in different stages of carcinogenesis.

The prognostic value of alpha-B-crystallin in human cancers has been addressed in few studies. In 2004, Chelouche-Lev et al. analyzed the expression of alpha-B-crystallin in human breast carcinomas and found a strong association between the expression of alpha-Bcrystallin and the presence of lymph node metastasis. ${ }^{18}$ Moyano et al. in 2006 reported that the expression of alpha-B-crystallin is correlated with poor prognosis of breast cancer. ${ }^{10}$ In a study of HNSCC, Chin et al. in 2004 reported that in all patients who lack the expression of alpha-B-crystallin, no recurrence was observed. ${ }^{3}$ This suggests that alpha-B-crystallin has a potential prognostic value. ${ }^{11}$ However, the present study on a large number of patients did not confirm the prognostic role of alpha-B-crystallin with outcome of patients with HNSCC. There was no correlation found with either disease-free or overall survival. Furthermore, the expression of alpha-B-crystallin was not significantly different in the group of patients with recurrences in comparison to patients who remained disease free.

Previous studies on the relationship between p53 expression and prognosis vary in different populations, reporting either a correlation with overall survival or with relapse or no correlation at all ${ }^{19-22}$. In this study grade three p53 expression was found to be significant with a shorter overall survival in comparison with lower grade p53 expression.

In conclusion, the expression of both p53 and alpha-B-crystallin is related to the differentiation and the location of the HNSCC. In this study p53 expression has a high prevalence in HNSCC and could therefore be a target for gene therapy. The role of the alphaB-crystallin in tumourigenesis and its interaction with other proteins is still not clear. There is lack of correlation between alpha-B-crystallin expression and the outcome of patients. 
Nevertheless, the presence of alpha-B-crystallin in a proportion of HNSCC implies that it has a role in the pathogenesis of HNSCC. 


\section{REFERENCES}

1 Parkin DM1, Bray F, Ferlay J, Pisani P. Global Cancer Statistics, 2002. CA Cancer J Clin 2005;55:74-108.

2. Carvalho AL, Nishimoto IN, Califano JA, Kowalski LP. Trends in incidence and prognosis for head and neck cancer the United States: a site-specific analysis of the SEER database. Int J Cancer 2005;114:806-816.

3. Chin D, Boyle GM, Theile DR, Parsons PG, Coman WB. Molecular introduction to head and neck cancer (HNSCC) carcinogenesis. Br J Plast Surg 2004;57:595-602

4. Thomas GR, Nadiminti H, Regalado J. Molecular predictors of clinical outcome in patients with head and neck squamous cell carcinoma. Int J Exp Pathol. 2005;86:347-363

5. Bauer JH, Helfand SL. New tricks of an old molecule: lifespan regulation by p53. Aging Cell. 2006;5:437-440

6. Greenblatt MS, Bennet WP, Hollstein M, Harris CC. Mutations in the p53 tumour suppressor gene: clues to cancer etiology and molecular pathogenesis. Cancer Res 1994; $54: 4855-878$.

7. Geisler SA, Olshan AF, Weissler MC, Cai J, Funkhouser WK, Smith J, Vick K. p16 and p53 protein expression as prognostic indicators of survival and disease recurrence from head and neck cancer. Clin Cancer Res. 2002;8:3445-3453.

8. Schmitt E, Brunet M, Hammann A, Solary E, Garrido C. Small heat shock proteins HSP27 and alpha-B-crystallin: cytoprotective and oncogenic functions. Antioxid Redox Signal. $2005 ; 7: 404-413$.

9. Gruvberger-Saal SK, Parsons R. Is the small heat shock protein alpha-B-crystallin an oncogene? J Clin Invest. 2006;116:30-32.

10. Moyano JV, Evans JR, Chen F, et al. alpha-B-crystallin is a novel oncoprotein that predicts poor clinical outcome in breast cancer. J Clin Invest. 2006;116:261-270. 
11. Chin D, Boyle GM, Williams RM, et al. alpha-B-crystallin, a new independent marker for poor prognosis in head and neck cancer. Laryngoscope. 2005;115:1239-1242.

12. Kamradt MC, Lu M, Werner ME, et al. The small heat shock protein alpha B-crystallin is a novel inhibitor of TRAIL-induced apoptosis that suppresses the activation of caspase-3. J Biol Chem. 2005;280:11059-1166.

13. Cardesa A, Gale N, Nadal A, Zidar N. Squamous cell carcinoma. In: World Health Organization classification of of Tumours. Barnes L, Eveson JE, Sidransky D (eds). Pathology and genetics, head and neck tumours. IARC Press, Lyon (France), 2005, pp118123.

14. Stuart K, Md Abdul Khaleque, Douglas B. Swayer, Ciocca DR. Heat shock protein: chaperones of tumorigenesis. Trends Biochem Sci. 2006;31:164-172.

15. Liu S, Li J, Tao Y, Xiao X. Small heat shock protein alphaB-crystallin binds to p53 to sequester its translocation to mitochondria during hydrogen peroxide-induced apoptosis. Biochem Biophys Res Commun. 2007;354:109-114.

16. Nishioka H, Hiasa Y, Hayashi I, Kitahori Y, Konishi N, Sugimura M. Immunohistochemical detection of p53 oncoprotein in human oral squamous cell carcinomas and leukoplakias: comparison with proliferating cell nuclear antigen staining and correlation with clinicopathological findings. Oncology 1993;50:426-429

17. Carlos de Vicente J, Junquera Gutierrez LM, Zapatero AH, et al. Prognostic significance of p53 expression in oral squamous cell carcinoma without neck node metastases. Head Neck. 2004;26:22-30.

18. Chelouche-Lev D, Kluger HM, Berger AJ, Rimm DL, Price JE. Alpha B-crystallin as a marker of lymph node involvement in breast cancer. Cancer 2004;100:2543-2548. 
19. Koch WM, Brennan JA, Zahurak M, Goodman SN, Westra WH, Schwab D, Yoo GH, Lee DJ, Forastiere AA, Sidransky D. p53 mutation and locoregional treatment failure in head and neck squamous cell carcinoma. J Natl Cancer Inst 1996;88:1580-1586.

20. Geisler SA, Olshan AF, Weissler MC, Cai J, Funkhouser WK, Smith J, Vick K. p16 and p53 protein expression as prognostic indicators of survival and disease recurrence from head and neck cancer. Clin Cancer Res. 2002; 8:3445-3453.

21. Mineta H, Borg A, Dictor M, Wahlberg P, Akervall J, Wennerberg J. p53 mutation, but not p53 overexpression, correlates with survival in head and neck squamous cell carcinoma. Br J Cancer 1998; 78:1084-1090.

22. Warnakulasuriya S, Jia C, Johnson N, Houghton J. P53 and P-glycoprotein expression are significant prognostic markers in advanced head and neck cancer treated with chemo/radiotherapy. J Pathol 2000; 191:33-38 


\section{Figure Legends}

Figure 1. p53 staining in HNSCC showing the p53 staining in the periphery of the tumour cell nests.

Figure 2. Alpha B-crystallin staining in HNSCC showing the Alpha B-crystallin staining in the centre of the tumour cell nests.

Figure 3. Survival curve of the patients with HNSCC showing the tumours with grade 3 expression of p53 ( $\geq 70 \%$ tumour stained up) has a poorer overall survival than the other patients. 
Table 1. Clinicopathological parameters of p53 positive and negative HNSCC

Parameters

$$
\text { p53 positive }
$$

$(\mathrm{n}=73)$

p53 negative

$(\mathrm{n}=45)$

Male: female

$52: 21$

$36: 9$

$\mathrm{p}=0.385$

Mean age (years) 63

60

$\mathrm{p}=0.221$

Grade

Well

Moderate

$21(28.8 \%)$

$20(44.4 \%)$

$\mathbf{p}=\mathbf{0 . 0 5}$

Poor

$42(57.5 \%)$

$24(53.4 \%)$

$10(13.7 \%)$

$1(2.2 \%)$

\section{Location}

Oral cavity

Oropharynx

$27(37.0 \%)$

$21(28.8 \%)$

$11(15.1 \%)$

$14(19.1 \%)$

$14(31.1 \%)$

$11(24.4 \%)$

$6(13.4 \%)$

Larynx

$$
14(19.1 \%)
$$$$
14(31.1 \%)
$$

significant test

\section{Lymph node \\ metastases}

Positive

$30(41.0 \%)$

Negative

$43(59 \%)$

$20(44.4 \%)$

$p=0.721$

Distant metastases

Positive

Negative

$72(98.6 \%)$

$1(1.4 \%)$

$44(97.7 \%)$

$1(2.3 \%)$

\section{TNM Stage}

$\begin{array}{llcl}\text { I } & 16(21.9 \%) & 11(24.4 \%) & \mathrm{p}=0.442 \\ \text { II } & 16(21.9 \%) & 8(17.8 \%) & \\ \text { III } & 9(12.3 \%) & 10(22.2 \%) & \\ \text { IV } & 32(43.9 \%) & 16(35.6 \%) & \end{array}$


Table 2. Clinicopathological parameters of alpha B-crystallin positive and negative HNSCC

\begin{tabular}{|c|c|c|c|}
\hline Parameters & $\begin{array}{l}\text { alpha B-crystallin } \\
\text { positive } \\
(\mathrm{n}=33)\end{array}$ & $\begin{array}{l}\text { alpha B-crystallin } \\
\text { negative } \\
(\mathrm{n}=85)\end{array}$ & significant test \\
\hline Male: female & $27: 6$ & $61: 24$ & $\mathrm{p}=0.683$ \\
\hline Mean age (years) & 62 & 61 & $\mathrm{p}=0.348$ \\
\hline \multicolumn{4}{|l|}{ Grade } \\
\hline Well & $11(33.3 \%)$ & $30(35.5 \%)$ & $p=0.179$ \\
\hline Moderate & $21(63.3 \%)$ & $45(52.9 \%)$ & \\
\hline Poor & $1(3.0 \%)$ & $10(11.8 \%)$ & \\
\hline
\end{tabular}

\title{
Location
}

Oral cavity

Oropharynx

$13(39.4 \%)$

$28(33.0 \%)$

$\mathrm{p}=0.249$

Hypopharynx

$6(18.2 \%)$

$26(30.6 \%)$

$6(18.2 \%) \quad 11(12.9 \%)$

Larynx

$8(24.2 \%)$

$20(23.5 \%)$

\section{Lymph node}

metastases

Positive

$14(42.4 \%)$

$19(57.6 \%)$

$36(42.4 \%)$

$\mathrm{p}=0.994$

Negative

$49(57.6 \%)$

Distant metastases

Positive

Negative

$32(96.7 \%)$

$84(98.8 \%)$

$1(1.2 \%)$

$\mathrm{p}=0.483$

\section{TNM Stage}

I

II

$1(3.3 \%)$

\author{
$1(1.2 \%)$
}

$\begin{array}{lcl}6(18.2 \%) & 21(24.7 \%) & \mathrm{p}=0.837 \\ 9(27.3 \%) & 15(17.6 \%) & \\ 4(12.1 \%) & 15(17.6 \%) & \\ 14(42.4 \%) & 34(40.1 \%) & \end{array}$


Figure 1

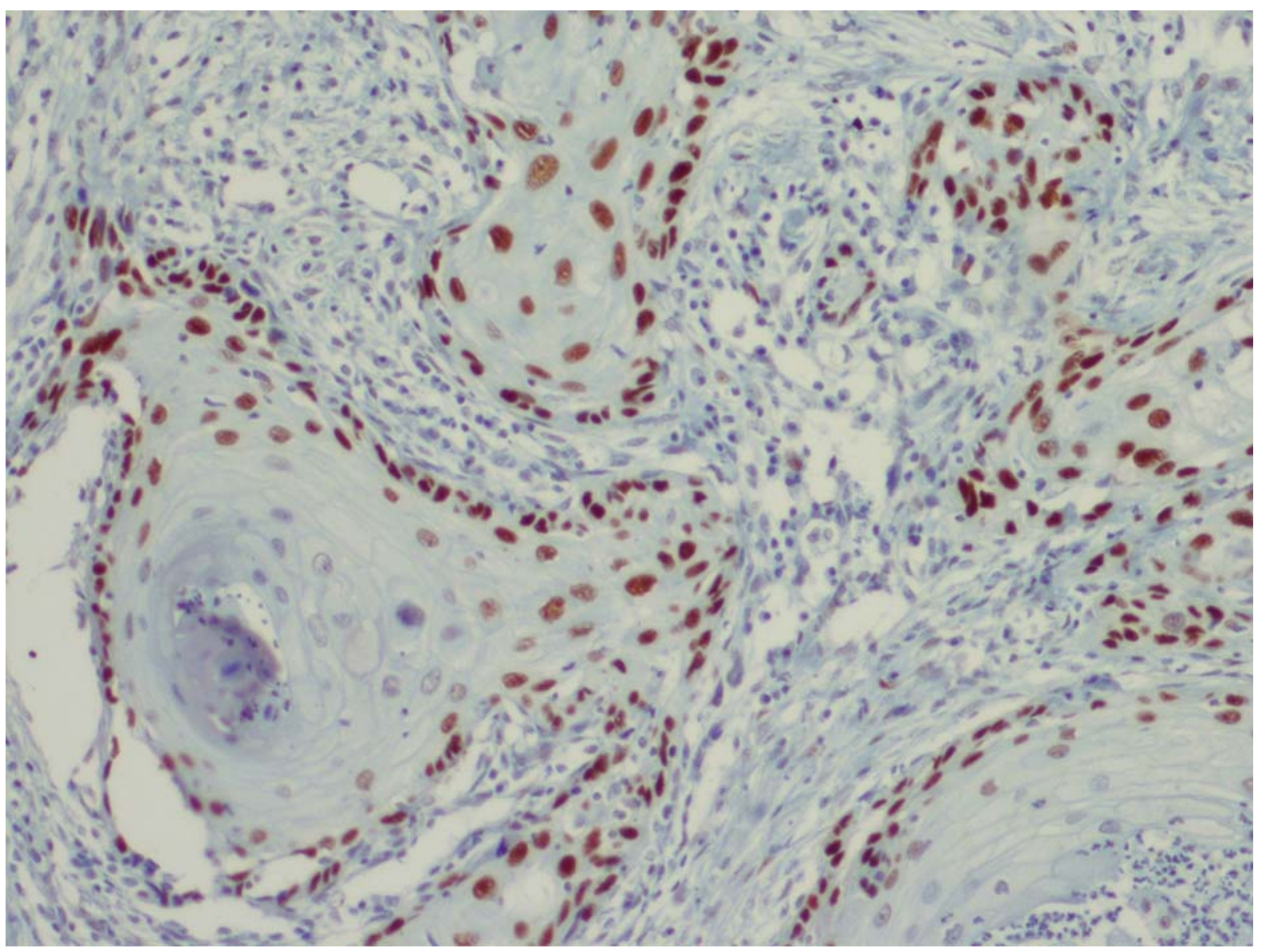


Figure 2

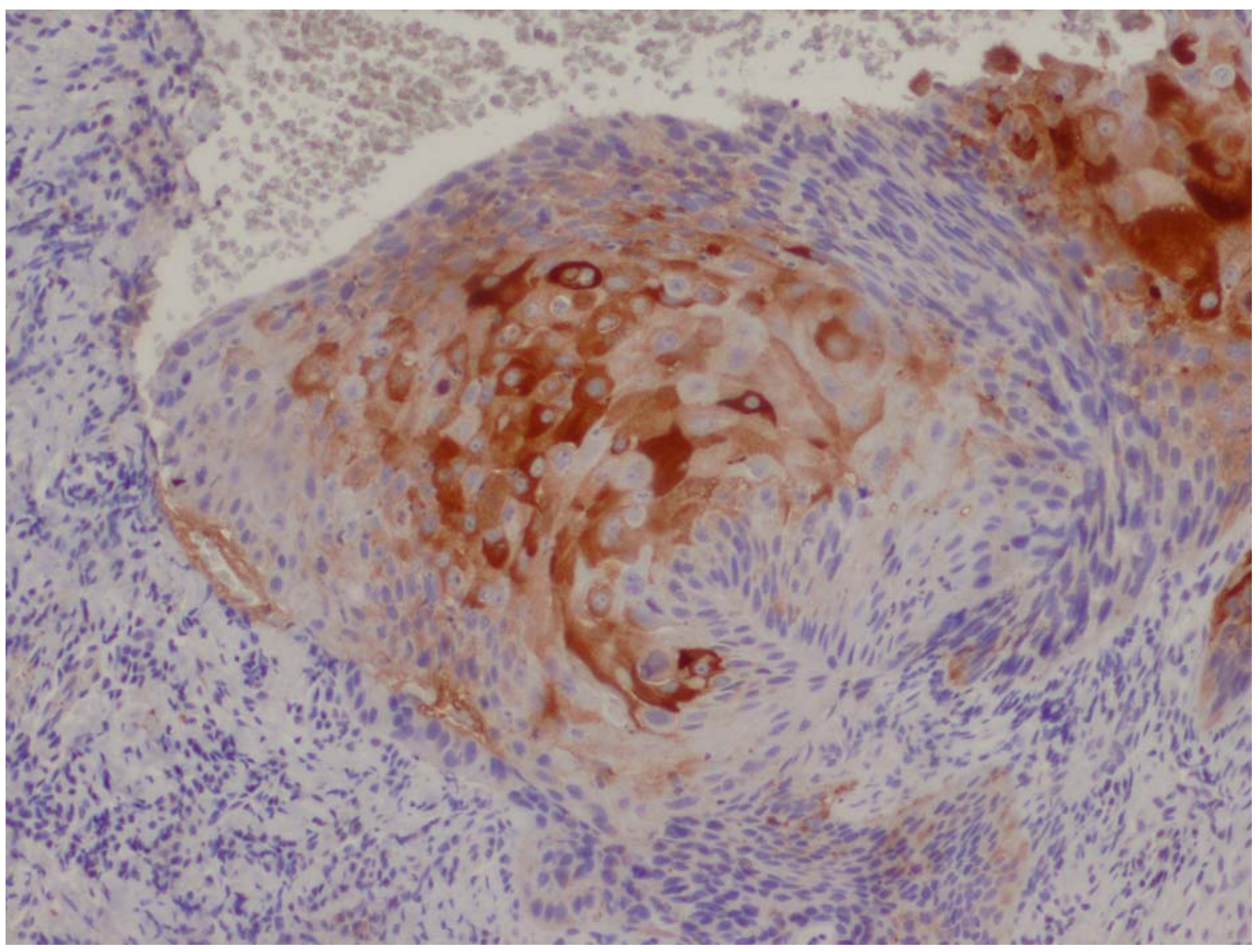


Figure 3

P53 grading and patient survival

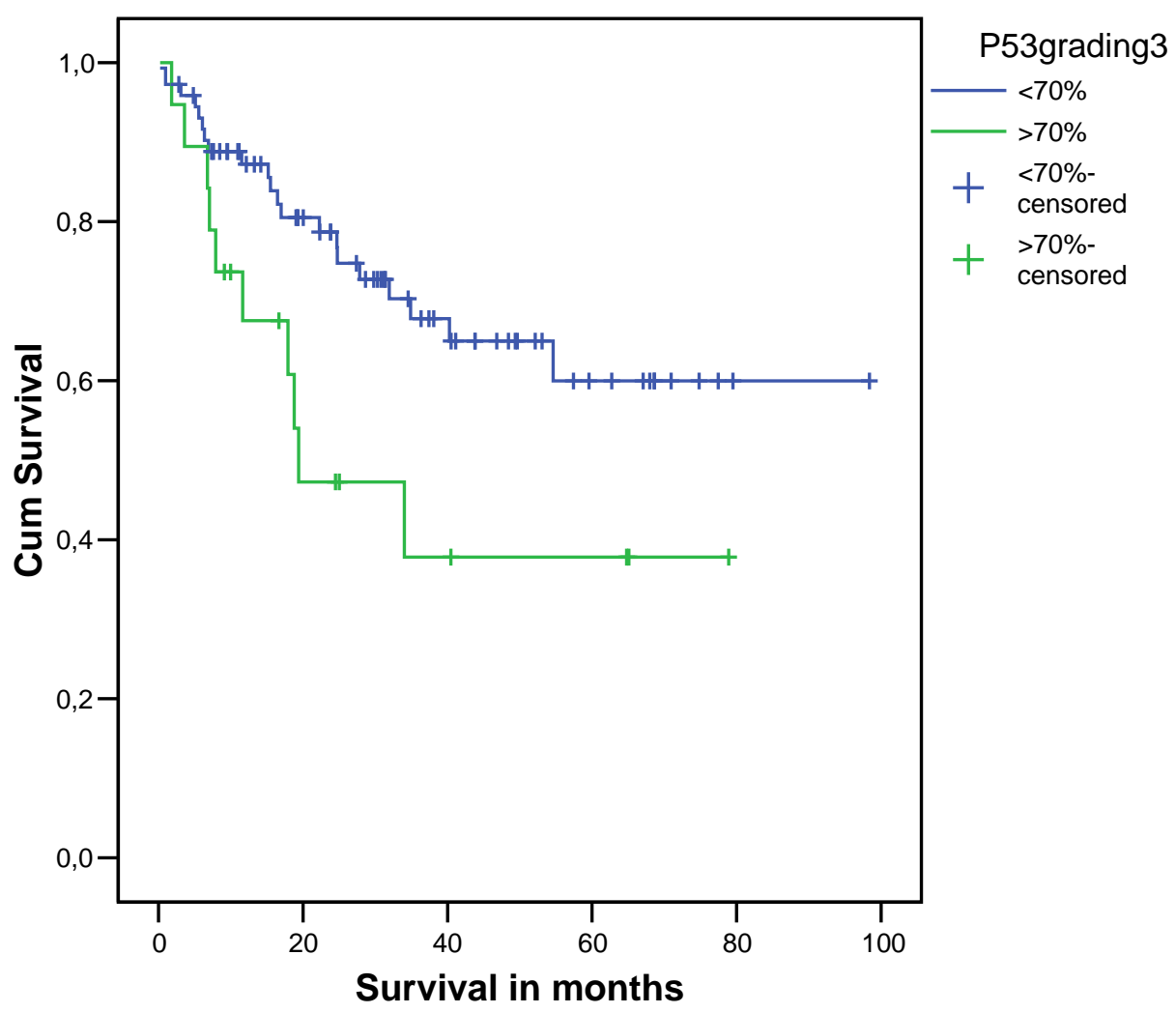

